\title{
Trends in Population Dynamics of Escherichia coli Sequence Type 131, Calgary, Alberta, Canada, 2006-2016
}

\author{
Gisele Peirano, ${ }^{2}$ Tarah Lynch, ${ }^{2}$ Yasufumi Matsumara, ${ }^{2}$ Diego Nobrega, \\ Thomas J. Finn, Rebekah DeVinney, Johann D.D. Pitout
}

Global expansion of antimicrobial drug-resistant Escherichia coli sequence type (ST) 131 is unrivaled among human bacteria. Understanding trends among ST131 clades will help with designing prevention strategies. We screened $E$. coli from blood samples ( $n=1,784)$ obtained in Calgary, Alberta, Canada, during 2006, 2012, and 2016 by PCR for ST131 and positive samples ( $\mathrm{n}=$ 344) underwent whole-genome sequencing. The incidence rate per 100,000 residents increased from 4.91 during 2006 to 12.35 during 2012 and 10.12 during 2016. ST131 belonged to clades A (10\%), B (9\%), and C (81\%). Clades C1-nonM27 and B were common during 2006, and C2 containing bla ${ }_{\mathrm{CTX}-\mathrm{M}-15}, \mathrm{C} 1-\mathrm{M} 27$ containing bla ${ }_{\text {СTX- }}$ ${ }_{M-27}$, and $A$ were responsible for the increase of ST131 during 2012 and 2016. C2 was the most antimicrobial drug-resistant subclade and increased exponentially over time. Eradicating ST131, more specifically the C2 subclade, will lead to considerable public health benefits for persons in Calgary.

Fscherichia coli sequence type (ST) 131 is the quintEessential example of a successful, global, antimicrobial-resistant, high-risk clone among human bacteria $(1,2)$. Currently, ST131 is the most common global extraintestinal pathogenic E. coli (ExPEC) clone; up to $30 \%$ of all ExPEC, $60 \%-90 \%$ of fluoroquinolone-resistant ExPEC, and $40 \%-80 \%$ of ExPEC with extended-spectrum $\beta$-lactamases [ESBLs] belong to

Author affiliations: University of Calgary Cummings School of Medicine, Calgary, Alberta, Canada (G. Peirano, T. Lynch,

T.J. Finn, R. De Vinney, J.D.D. Pitout); Alberta Precision

Laboratories, Calgary (G. Peirano, T. Lynch, J.D.D. Pitout); Kyoto

University Graduate School of Medicine, Kyoto, Japan

(Y. Matsumara); University of Calgary, Calgary (D. Nobrega);

University of Pretoria, Pretoria, South Africa (J.D.D. Pitout)

DOI: https://doi.org/10.3201/eid2612.201221
ST131 $(3,4)$. Population genetics indicate that ST131 consists of different clades (5): clade A contains serotype O16:H5 and fim H41, clade B contains mostly serotype $\mathrm{O} 25 \mathrm{~b}: \mathrm{H} 4$ and fim $\mathrm{H} 22$, and clade $\mathrm{C}$ contains serotype $\mathrm{O} 25 \mathrm{~b}: \mathrm{H} 4$ and fim $\mathrm{H} 30$. Clade $\mathrm{C}$ is divided into 2 subclades: C1/H30R (associated with fluoroquinolone resistance) and C2/H30Rx (associated with fluoroquinolone resistance and $\left.b l a_{\mathrm{CTX}-\mathrm{M}-15}\right)$. A novel ST131 C1 subclade, known as C1-M27 with bla $a_{\text {Стх-м-27 }}$ was reported in Japan (6).

ST131 is the most dominant and most antimicrobial-resistant among E. coli causing bloodstream infections in Calgary, Alberta, Canada, infecting mostly the elderly in long-term care centers (7). Previous molecular epidemiology studies from the same region showed that ST131 was relatively rare among ESBL-producing and fluoroquinoloneresistant $E$. coli during the early 2000s but showed a major increase toward the end of the 2000s $(8,9)$. However, limited information is available regarding the changes in population dynamics of ST131 clades over extended periods, especially among nonbiased E. coli isolates in large, well-defined, geographic regions.

To address this issue, we conducted a retrospective cohort study that characterized ST131 clades responsible for bloodstream infections in Calgary over an 11-year period (2006-2016). Investigating trends of ST131 clades over long periods by using a populationbased surveillance approach will aid in clarifying the evolution of this clone and help with designing superior prevention strategies $(3,10)$.

\footnotetext{
${ }^{1}$ Accepted as an oral presentation for the 30th European Congress of Clinical Microbiology and Infectious Diseases, Paris, France, April 18-21, 2020.
}

${ }^{2}$ These authors contributed equally to this article. 


\section{Materials and Methods}

\section{Study Population}

We conducted a retrospective cohort study in Calgary by using all $E$. coli human clinical isolates from blood cultures processed by a centralized laboratory system (Alberta Precision Laboratories) during 2006, 2012, and 2016. All blood culture samples from adults and children in inpatient and outpatient settings were included.

\section{Clinical Data}

Clinical information corresponding to source patients at the time of the E. coli bloodstream infection was obtained by using Sunrise Clinical Manager (Allscripts Healthcare Solutions, Inc., https://www.allscripts. com). A case-patient with an E. coli bloodstream infection was defined as a patient with systemic inflammatory response and documented growth of an $E$. coli isolate in a blood culture. Incident case-patients were defined as Calgary residents with a first isolation of E. coli from blood. Repeat E. coli from blood were excluded. Bloodstream infections were defined as community acquired, hospital acquired, or healthcare associated (11).

\section{Bacterial Isolates, Identification, and Susceptibility Testing}

All E. coli isolates from blood were routinely stored at Alberta Precision Laboratories and available for this study. Unique isolates recovered during January 1-December 31, 2006, 2012, and 2016 were obtained from the frozen depository.

Identification was conducted by using matrix-assisted laser desorption/ionization time-of-flight mass spectrometry (Vitek; bioMérieux, https://www.biomerieux.com), and susceptibility testing was conducted the VITEK 2 Instrument (bioMérieux). Susceptibilities were determined for amoxicillin/clavulanic acid, piperacillin/tazobactam, ceftriaxone, meropenem, ertapenem, amikacin, gentamicin, tobramycin, ciprofloxacin, and trimethoprim/sulfamethoxazole. Throughout this study, results were interpreted by using the Clinical Laboratory Standards Institute criteria for broth dilution (12). Antimicrobial resistance and virulence scores were determined as described (13).

\section{Molecular Characterization}

All E. coli isolates $(n=1,786)$ were initially screened with a PCR specific for ST131 (14). Positive isolates $(n=344)$ underwent whole-genome sequencing, by using procedures previously $(15,16)$. The Nextera XT DNA Sample Preparation Kit (Illumina, https:/ / www.illumina.com) was used to prepare libraries for sequencing. Samples were multiplexed and sequenced on an Illumina NextSeq500 for 300 cycles (151-bp paired-end). Draft genomes were obtained by using SPAdes version 3.10.1 (17). To define the presence of genes and mutations, BLAST (18) in combination with following databases or typing schemes were accessed: National Center for Biotechnology Information Bacterial Antimicrobial Resistance Reference Gene Database (https://www.ncbi.nlm.nih. gov/bioproject/PRJNA313047), ResFinder (19), PlasmidFinder (20), MLST (21) virulence finder (22), and virulence factor database (23). ST131 clades were identified by using an in silico PCR and primers described elsewhere (14).

\section{Statistical Analysis}

The Fisher exact test was used to perform pairwise comparisons of factors between clades, $t$-test was used for age comparisons, and $\mathrm{p}$ values obtained within individual categories were adjusted for multiple comparisons by using the false discovery rate (24). Population data were extracted from census reports from Statistics Canada (https://www.statcan.gc.ca) and used to estimate incidence rates (IRs) on the basis of a Poisson distribution. The MannWhitney test was used to compare antimicrobial resistance and virulence scores between clades. The effect of eliminating subclade C2 on nonsusceptibility and IRs was assessed by using Fisher exact and Poisson tests, for which population characteristics were compared with the presence and absence of subclade C2 isolates. The $p$ values were adjusted for multiple comparisons accordingly. All analyses were conducted in $\mathrm{R}$ version 3.6.1 (25). Statistical significance was set at the $5 \%$ level.

\section{Sequence Data Accession Numbers and Ethics}

Sequencing data was deposited in the National Center for Biotechnology Information database (submission no. SUB7225977). This study was approved by the University of Calgary Conjoint Health Research Ethics Board (REB16-2457).

\section{Results}

\section{E. coli Isolates}

E. coli was the most common bacterium obtained from blood in the Calgary region during 2006 (482 [28.9\%] of 1,669 isolates), 2012 (691 [29.7\%] of 2,084 isolates), and 2016 (685 [31.1\%] of 2,201 isolates). A total of 1,786 unique $E$. coli were screened for ST131: 481 from 2006, 621 from 2012, and 684 from 2016. Overall, $344(19.2 \%)$ of 1,786 E. coli isolates were PCR positive 
for ST131; the prevalence of ST131 increased from 53 (11\%) of 481 during 2006 to 150 (24.2\%) of 621 during 2012 and $141(20.6 \%)$ of 684 during 2016 ( $<<0.001$ for both comparisons).

Most ST131 isolates belonged to clade $\mathrm{C}$ in the following subclades (Table 1, https:/ / wwwnc.cdc.gov/ EID/article/26/12/20-1221-T1.htm): C0 ( $\mathrm{n}=5,2 \%)$, C1-nonM27 ( $\mathrm{n}=121,35 \%), \mathrm{C} 1-\mathrm{M} 27(\mathrm{n}=13,4 \%)$, and $\mathrm{C} 2(\mathrm{n}=139,40 \%)$. The remainder of ST131 isolates belonged to clades A $(n=34[10 \%)]$ and B $(n=32[9 \%])$.

\section{Incidence Rates and Population Dynamics of ST131 Clades}

The IR per 100,000 residents with ST131 bloodstream infections in Calgary increased from 4.91 during 2006 to 12.35 during 2012 and 10.12 during 2016 ( $<<0.001$ for both comparisons). Overall, the population structure of ST131 was dominated by the C clade. However, the IRs per 100,000 residents and proportions among the different subclades showed a major change over time (Table 2; Figure). The C0 subclade represented $9.4 \%$ of the ST131 population during 2006, with an estimated IR of 0.46 cases per 100,000 residents. However, the $\mathrm{C} 0$ subclade was not detected during 2012 and 2016 ( $\mathrm{p}=0.001$ for both comparisons).

The C1-nonM27 subclade dominated the population structure of ST131 during 2006 (comprising of 46\% of the total population, with an IR of 2.22/100,000 residents). Despite an increased IR during 2012 and 2016 (when compared with that for 2006), the frequency of C1-nonM27 isolates decreased to 37.3\% in 2012 and $29 \%$ in 2016 (2006 vs. 2016; $p=0.04$ ) (Figure). The C1M27 subclade increased from 1.9\% during 2006 to 5.7\% during 2016. There was an association between C1$\mathrm{M} 27$, the presence of $b l a_{\mathrm{CTX}-\mathrm{M}-27}$, and year of isolation (5 C1-M27 isolates from 2006 and 2012 were negative for $b l a_{\text {СTX-M-27 }}$ and 7/8 isolates obtained during 2016 were positive for bla $\left.{ }_{\mathrm{CTX}-\mathrm{M}-27}\right)(\mathrm{p}=0.004)$ (Figure).

The prevalence of $\mathrm{C} 2$ subclade increased substantially from $17 \%$ of the total ST131 population during 2006 to $42 \%$ during 2012 and $47 \%$ during 2016 ( $p<0.001$ for both comparisons) (Figure). The IR per
100,000 residents of the C2 clade increased from 0.83 during 2006 to 5.19 during 2012 and 4.81 during 2016 $(\mathrm{p}<0.001$ for both comparisons) (Table 2). The increase in subclade $\mathrm{C} 2$ correlated with the presence of CTX-M-15 (4 [44\%] of 9 of C2 isolates from 2006 were positive for $b l a_{\text {CTX-M-15 }}$ compared with 89 [68\%] of 130 isolates obtained during 2012 and 2016) (Figure).

Clade A was absent among ST131 during 2006 and then increased to $12 \%$ of the ST131 population during 2012 and $11.3 \%$ of the ST131 population during 2016 ( $p<0.01$ for both comparisons) (Figure). The IR of clade A increased from 0 to 1.48/100,001 residents during 2012 and to 1.15/100,000 residents during 2016 ( $p<0.001$ for both comparisons) (Table 2). B was the second most common clade during 2006 (26.4\% of the total ST131 population), but decreased to $6 \%$ of the ST131 population during 2012 and to $6.4 \%$ of the ST131 population during 2016 ( $\mathrm{p}<0.001$ for both comparisons) (Figure). The IR of clade B decreased from 1.30/100,000 residents to $0.74 / 100,000$ residents during 2012 and to 0.65/100,000 residents during 2016 (Table 2).

\section{Clinical Characteristics}

E. coli ST131 bloodstream infections were evenly distributed between male patients ( $\mathrm{n}=171,49.7 \%)$ and female patients $(n=173,50.3 \%)$ (Table 1). Just under half $(48 \%)$ of E. coli ST131 bloodstream infections were healthcareassociated, followed by community-acquired (34\%) and hospital-acquired (18\%) (Table 1). Clades A and B were associated with community-acquired infections, and patients infected with clade $\mathrm{C}$ were more likely to be healthcare associated. Patients infected with clade A tended to be younger (Table 1). More than half ( $\mathrm{n}=$ $186,54 \%$ ) of patients had upper urinary tract infections, followed by bloodstream infections with an unknown source $(n=69,20 \%)$, pneumonia $(n=35,10 \%)$, acute biliary tract infections $(\mathrm{n}=31,9 \%)$, and intraabdominal infections $(n=23,7 \%)$ (Table 1$)$.

\section{Serotypes, fimH Types, and Antimicrobial Susceptibilities}

Clade A contained O16:H5, fim $\mathrm{H} 41$, and fim $\mathrm{H} 89$. Clade B contained O25: $\mathrm{H} 4, \mathrm{O} 2: \mathrm{H} 4$, fim $\mathrm{H} 22$, fim $\mathrm{H} 27$,

\begin{tabular}{|c|c|c|c|}
\hline \multirow[b]{2}{*}{ Clade } & \multicolumn{3}{|c|}{ IR $(95 \% \mathrm{Cl})$} \\
\hline & 2006 & 2012 & 2016 \\
\hline A & $0.00^{\mathrm{a}}(0.00-0.34)$ & $1.48^{\mathrm{b}}(0.88-2.34)$ & $1.15^{\mathrm{b}}(0.66-1.87)$ \\
\hline B & $1.30(0.71-2.18)$ & $0.74(0.34-1.41$ & 0.65 (0.30-1.23) \\
\hline $\mathrm{CO}$ & $0.46^{a}(0.15-1.08)$ & $0.00^{\mathrm{b}}(0.00-0.30)$ & $0.00^{\mathrm{b}}(0.00-0.26)$ \\
\hline C1-nonM27 & $2.22^{\mathrm{a}}(1.55-3.60)$ & $4.61^{\mathrm{b}}(3.48-5.99)$ & $2.94^{\mathrm{a}}(2.11-3.99)$ \\
\hline C1-M27 & $0.09^{a}(0.00-0.56)$ & $0.33(0.09-0.84)$ & $0.57^{b}(0.25-1.13)$ \\
\hline $\mathrm{C} 2$ & $0.83^{a}(0.41-1.72)$ & $5.19^{b}(3.98-6.63)$ & $4.81^{b}(3.73-6.11)$ \\
\hline Total & $4.91^{\mathrm{a}}(3.68-6.42)$ & $12.35^{\mathrm{b}}(10.45-14.49)$ & $10.12^{\mathrm{b}}(8.52-11.94)$ \\
\hline
\end{tabular}

${ }^{*}$ Rates followed by different superscript letters indicate significant differences between years at the $5 \%$ level. IR; incidence rate. 


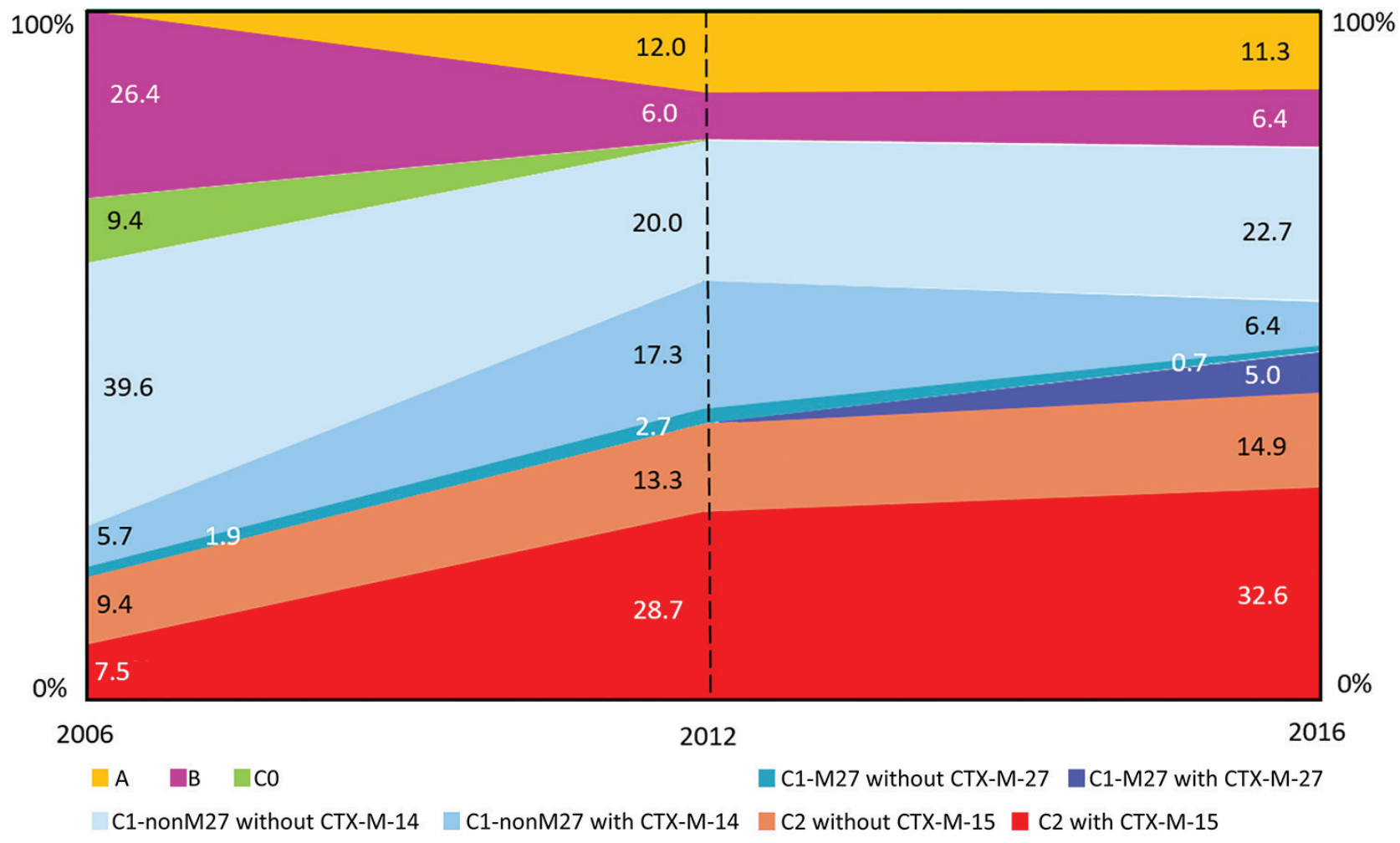

Figure. Proportions of Escherichia coli sequence type 131 clades, Calgary, Alberta, Canada, 2006-2016.

fim $\mathrm{H} 324$, and fim $\mathrm{H} 30$. Clade C contained O25:H4 and fim $\mathrm{H} 30$ (Table 1).

Overall, high $(>25 \%)$, intermediate, or resistant (not susceptible) rates were observed for ceftriaxone, ciprofloxacin, trimethoprim/sulfamethoxazole, gentamicin, and tobramycin. Low rates $(<5 \%)$ were observed for amikacin, ertapenem, and meropenem. C2 was the most antimicrobial-resistant subclade, followed by C1-nonM27 and C1-M27 (Table 1). Clades B and C0 were the most susceptible clades, and clade A showed high nonsusceptible rates for trimethoprim/sulfamethoxazole, gentamicin, and tobramycin (Table 1).

\section{Removal of Subclade C2}

Eliminating subclade C2 would have decreased the incidence rate of ST131 bloodstream infections from $12.35 / 100,000$ residents to $7.16 / 100,000$ residents during 2012 and from 10.12/100,001 residents to 5.31/100,000 residents during 2016 ( $\mathrm{p}<0.001$ both comparisons). In addition, eliminating subclade C2 would have resulted in a significant reduction of not susceptible rates for amoxicillin/clavulanic acid, ciprofloxacin, ceftriaxone, and tobramycin for ST131 causing bloodstream infections in Calgary (2006, 2012, and 2016) ( $\mathrm{p}<0.05$ for all comparisons).

\section{Quinolone Resistance-Determining Regions and Antimicrobial Resistance Determinants}

The combination of mutations in gyrase A genes ( $g y r A$ S83L and gyrA D87N) and DNA topoisomerase IV genes (parC S80I, parC E84V, and parE I529L) in the quinolone resistance-determining regions were present in all C1 and C2 isolates (Table 3). Nearly all (97\%) ST131 isolates contained the parE I529L mutation. Most (85\%) clade A isolates had the gyrA S83L mutation; for 5 isolates, this mutation was combined with gyrA D87N and parC S80I, and 1 isolate had the gyrA S83L, gyrA D87N, parC S80I, and parC E84V combination. Mutations in gyr $A$ and $\operatorname{par} C$ were rare in clade $B ; 2 / 32$ isolates had the gyrA S83L and parC S80I mutation combination (Table 3). One subclade $\mathrm{C} 0$ isolate had only the gyrA S83L mutation, and another $\mathrm{C} 0$ isolate had the gyrA S83L, gyrA $\mathrm{D} 87 \mathrm{~N}$, parC S80I, and parC E84V combination.

CTX-M $\beta$-lactamases were detected among 148 $(43 \%)$ isolates; most were CTX-M-15, followed by CTX-M-14, CTX-M-27, CTX-M-55, and CTX-M-198 (Table 3). CTX-M types were associated with different subclades (e.g., bla $a_{\text {CTX-M-14 }}$ with C1-nonM27, bla $a_{\text {CTX-M-15 }}$ with C2, bla $a_{\text {CTX-M-27 }}$ with C1-M27, and bla $a_{\text {CTX-M-55 }}$ with A). TEM-1 was common in most clades, with the exception of C2 and C1-M27. Three ST131 isolates were positive for $b l a_{\mathrm{CMY}-2^{\prime}}$ and $1 \mathrm{C} 2$ isolate was positive for $b l a_{\mathrm{NDM}-5^{*}}$. 
Certain aminoglycoside-modifying enzymes were common among ST131: aac(3)-IId, aac (6')-Ib$c r$, aadA5, aph(3'')-Ib, and aph (6)-Id (Table 3). Some associations between presences of aminoglycosidemodifying enzymes with certain subclades were noted: aac (3)-IId were present mainly in clades A, B, and C1-nonM27; aac (6')-Ib-cr in subclade C2; aadA2 in clade $\mathrm{B}$, and aadA5 in clades $\mathrm{A}, \mathrm{C} 0$, and $\mathrm{C} 1$. The combination of aph(3')-Ib and aph (6)-Id was more common in clades A and C1-nonM27 (Table 3). With regard to the presence of other antimicrobial resis- tance determinants, qnr was rare, and $d f r A 17$, sul1, sul2, and tetA were common among most of ST131 clades (Table 3).

\section{Plasmids and Replicon Types}

Overall, IncF plasmid types (e.g., combinations of FIA, FIB, FIC, and FII) were common among all ST131 clades. Col-like plasmids and other plasmid families (IncI1, IncN, IncX1, IncX4, and IncY) were widely distributed across all clades but less common than IncF types (Table 1).

Table 3. Factors associated with Escherichia coli sequence type 131 clades, Calgary, Alberta, Canada, 2006, 2012, and 2016*

\begin{tabular}{|c|c|c|c|c|c|c|c|}
\hline \multirow[b]{2}{*}{ Factor } & \multicolumn{7}{|c|}{ Clade } \\
\hline & $A, n=34$ & $\mathrm{~B}, \mathrm{n}=32$ & $\mathrm{C} 0, \mathrm{n}=5$ & C1-non-M27, n = 121 & C1-M27, $n=13$ & $C 2, n=139$ & All, $n=344$ \\
\hline \multicolumn{8}{|l|}{ QRDR mutation } \\
\hline gyrA S83L & $29(85)^{a, b}$ & $2(6)^{c}$ & $2(40)^{b, c}$ & $121(100)^{d}$ & $13(100)^{a, d}$ & $139(100)^{d}$ & $306(89)$ \\
\hline gyrA D87N & $5(15)^{\mathrm{a}}$ & $0^{\mathrm{a}}$ & $1(20)^{a}$ & $121(100)^{b}$ & $13(100)^{b}$ & $139(100)^{b}$ & $279(8)$ \\
\hline parC S80I & $5(15)^{a}$ & $2(6)^{a}$ & $1(20)^{\mathrm{a}}$ & $121(100)^{b}$ & $13(100)^{b}$ & $139(100)^{b}$ & $281(82)$ \\
\hline parC E84V & $1(3)^{a}$ & $0^{a}$ & $1(20)^{a}$ & $121(100)^{b}$ & $13(100)^{b}$ & $139(100)^{b}$ & $275(80)$ \\
\hline parE I529L & $30(88)^{a}$ & $27(84)^{a}$ & $5(100)$ & $121(100)^{b}$ & $13(100)$ & $139(100)^{b}$ & 335 (97) \\
\hline \multicolumn{8}{|l|}{$\beta$-lactamase } \\
\hline CTX-M-15 & $2(6)^{a}$ & $1(3)^{a}$ & $0^{\mathrm{a}}$ & $1(1)^{a}$ & $0^{\mathrm{a}}$ & $93(67)^{b}$ & $97(28)$ \\
\hline CTX-M-14 & $0^{a}$ & $0^{a}$ & 0 & $38(31)^{b}$ & 0 & $1(1)^{a}$ & $39(11)$ \\
\hline CTX-M-27 & $1(3)^{a}$ & $0^{\mathrm{a}}$ & 0 & $0^{\mathrm{a}}$ & $7(54)^{b}$ & $0^{a}$ & $8(2)$ \\
\hline CTX-M-55 & $2(6)$ & 0 & 0 & 0 & 0 & 0 & $2(0.6)$ \\
\hline CTX-M-198 & 0 & 0 & 0 & $1(1)$ & 0 & 0 & $1(0.3)$ \\
\hline NDM-5 & 0 & 0 & 0 & 0 & 0 & $1(1)$ & $1(0.3)$ \\
\hline OXA-1 & $0^{\mathrm{a}}$ & $0^{\mathrm{a}}$ & $0^{\mathrm{a}}$ & $1(1)^{a}$ & $0^{\mathrm{a}}$ & $84(6)^{b}$ & $85(25)$ \\
\hline OXA-9 & 0 & 0 & 0 & 0 & 0 & $1(1)$ & $1(0.3)$ \\
\hline SHV-12 & 0 & 0 & 0 & 0 & 0 & $2(1)$ & $2(0.6)$ \\
\hline TEM-1 & $29(85)^{a, b}$ & $20(63)^{\mathrm{a}}$ & $4(80)^{a, b}$ & $103(85)^{b}$ & $1(8)^{c}$ & $26(19)^{c}$ & $183(53)$ \\
\hline TEM other & 0 & 0 & 0 & $2(2)$ & 0 & $2(1)$ & $4(1)$ \\
\hline CMY-2 & 0 & $2(6 \%)$ & 0 & $1(1)$ & 0 & 0 & $3(0.9)$ \\
\hline \multicolumn{8}{|c|}{ Aminoglycoside-modifying enzyme } \\
\hline $\operatorname{Aac}(3)-I / a$ & $0^{a}$ & $0^{a}$ & 0 & $2(1)^{a}$ & $0^{a}$ & $57(41)^{\mathrm{b}}$ & $59(17)$ \\
\hline$A a c(3))-I I d$ & $11(32)^{a, b}$ & $15(47)^{a}$ & 0 & $63(52)^{a}$ & $0^{b, c}$ & $4(3)^{c}$ & $93(27)$ \\
\hline$a a c\left(6^{\prime}\right)-I b-c r$ & $0^{a}$ & $0^{a}$ & $0^{a}$ & $2(2)^{a}$ & $0^{\mathrm{a}}$ & $84(60)^{\mathrm{b}}$ & $86(25)$ \\
\hline aadA1 & 0 & $26)$ & 0 & 0 & 0 & $4(3)$ & $6(2)$ \\
\hline aadA16 & 0 & 0 & 0 & $1(1)$ & 0 & $1(1)$ & $2(0.6)$ \\
\hline aadA2 & $0^{\mathrm{a}}$ & $14(44)^{b}$ & 0 & $0^{\mathrm{a}}$ & $0^{a}$ & $2(1)^{a}$ & 16() \\
\hline $\operatorname{aad} A 5$ & $23(68)^{a}$ & $1(3)^{b}$ & $3(60)^{a, c}$ & $70(58)^{a}$ & $7(54)^{a, c}$ & $57(41)^{c}$ & $161(47)$ \\
\hline ant(2")-la & 0 & 0 & 0 & 0 & 0 & $5(4)$ & $5(1)$ \\
\hline$a p h\left(3^{\prime}\right)-l a$ & $1(3)$ & 2 & 0 & $1(11)$ & 0 & 0 & $4(1)$ \\
\hline$a p h\left(3^{\prime \prime}\right)-l b$ & $20(59)^{a}$ & $3(9)^{b}$ & 0 & $69(57)^{a}$ & $5(38)$ & $26(19)^{b}$ & $123(36)$ \\
\hline$a p h\left(3^{\prime}\right)-11 a$ & 0 & $1(3)$ & 0 & $1(1)$ & 0 & 0 & $2(0.6)$ \\
\hline$A p h(6)-I c$ & 0 & $1(3)$ & 0 & $1(1)$ & 0 & 0 & $2(0.6)$ \\
\hline$A p h(6)-I d$ & $20(59)^{a}$ & $3(9)^{b}$ & 0 & $68(56)^{a}$ & $5(38)$ & $25(18)^{b}$ & $121(35)$ \\
\hline \multicolumn{8}{|l|}{ Other } \\
\hline qnrB & 0 & 0 & 0 & 0 & 0 & $2(1)$ & $2(0.6)$ \\
\hline$A R R-3$ & 0 & 0 & 0 & $1(1)$ & 0 & 1 (1) & $2(0.6)$ \\
\hline dfrA1 & 0 & $1(3)$ & 0 & 0 & 0 & 0 & $1(0.3)$ \\
\hline dfrA12 & $0^{\mathrm{a}}$ & $13(41)^{b}$ & 0 & $0^{a}$ & $0^{\mathrm{a}}$ & $2(1)^{a}$ & $15(4)$ \\
\hline dfrA14 & $1(3)$ & 0 & 0 & 0 & 0 & 8 & $12(3)$ \\
\hline dfrA17 & $22(65)^{a}$ & $1(3)^{b}$ & $3(60)^{a}$ & $70(58)^{a}$ & $7(54)^{\mathrm{a}}$ & $59(42)^{a}$ & $162(47)$ \\
\hline dfrA27 & 0 & 0 & 0 & $1(1)$ & 0 & $1(1)$ & $2(0.6)$ \\
\hline dfrA5 & 0 & $1(3)$ & 0 & 0 & 0 & 0 & $1(0.3)$ \\
\hline sul1 & $22(65)$ & $15(47)$ & $3(60)$ & 69 (57) & $6(46)$ & $63(45)$ & $178(52)$ \\
\hline sul2 & $20(59)^{a}$ & $3(9)^{b}$ & 0 & $70(58)^{a}$ & $5(38)$ & $28(20)^{b}$ & $126(37)$ \\
\hline sul3 & 0 & $1(3)$ & 0 & 0 & 0 & 0 & $1(0.3)$ \\
\hline tetA & $19(56)^{a}$ & $5(16)^{b}$ & 0 & $61(50)^{a}$ & $6(4)$ & $77(55)^{a}$ & $168(49)$ \\
\hline tet $B$ & $2(6)$ & $3(9)$ & 0 & $2(2)$ & 0 & $3(2)$ & $10(3)$ \\
\hline
\end{tabular}

*Values are no. (\%). Rates followed by different superscript letters indicate significant differences between clades at the $5 \%$ level (adjusted for multiple comparisons). 
Using IncF plasmid replicons (FII_1, FIA_2, FIB_20, FII_2, and FIA_1) and a plasmid classification system published recently (26), we found that group 1 plasmids (combination of FII_1, FIA_2, and FIB_20) were in clades A, B, C1-nonM27, and C1-M27, and group 2 plasmids (combination of FII_2 and FIA_1) were in clades $\mathrm{C} 0$ and $\mathrm{C} 2$ (Table 1). Group 1 plasmids were common among $\mathrm{C} 1$ clades, and group 2 plasmids were common among $\mathrm{C} 2$ isolates.

\section{Virulence-Associated Factors}

The presence of 37 putative virulence factors were assessed for different clades (Table 4). The following factors were present among most isolates: papAIX, iha, fimH, sat, fyuA, usp, iss, and malX. Some virulence factors were associated with certain clades: papBCFJK, iha, hlyA, and cnf1 with subclade C2; af$a A B C D$, draABCDP vat, and traT with clade $\mathrm{A}$; af$a A B C D$, dra $A B C D P, k p s M I I$, and $i b e A B C$ with clade $\mathrm{B}$; and kpsMTIII with subclades $\mathrm{C} 0$ and $\mathrm{C} 1$. No major differences in virulence scores were observed for the different clades.

\section{Discussion}

The abrupt global expansion of ST131 during the 2000s is unrivaled among human bacteria and is a real-world model for the evolution of antimicrobial-resistant high-risk clones (10). This study describes the clinical features, incidence rates, genomic characteristics, and changes in population structure of ST131 clades causing bloodstream infections in a large centralized region of Canada over an 11-year period (2006-2016). The incidence rates and prevalence of ST131 increased over the time period, mostly caused by an influx of subclades C2 with bla $a_{\text {СТХ-M-15 }}$ and C1-M27 with bla $a_{\text {CTX-M-27. }}$. Such results reinforce the possible role of CTX-M enzymes in the evolutionary success of ST131 (10). The presence of $b l a_{\text {СТХ-M-14 }}$ among C1-nonM27 isolates did not provide a beneficial advantage to this subclade. This finding is probably caused by clonal interference among 2 clones that have acquired different beneficial mutations competing in the same environment (27).

The population structure of ST131 in the Calgary region was dominated by clade $\mathrm{C}$, which is similar to results from a previous large global study (28). The C clade originated from clade B during the mid to late 1980 s by acquisition of several prophages, genomic islands, the fimH3O allele, and mutations within gyrA and parC that likely transpired in North America $(29,30)$. The $C$ clade in this study was mostly responsible for healthcare-associated urinary tract infections. C2 was the most common and most antimicrobial-resistant subclade in this collection and was associated with group 2 plasmids, $b l a_{\text {СТХ-M-15 }}$ and $a a c\left(6^{\prime}\right)-I b-c r$, as well as the virulence factors iha, hlyA, and cnf1. This subclade became prominent during 2012 and 2016 and showed the highest IRs among all subclades during this period. The increase of C2 correlated with the presence of CTX-M-15. Elimination of the $\mathrm{C} 2$ subclade through vaccination or phagetherapy programs (31), will lead to major decreases in incidence and antimicrobial-resistant rates among ST131 causing bloodstream infections in Calgary.

The C1-nonM27 subclade was the most common subclade during 2006 and associated with group 1 plasmids, bla $a_{\text {СТХ-M-14' }}$ and aac (3)-IId. Overall, the C1M27 subclade was rare (especially during 2006 and 2012) but increased substantially during 2016, which correlated with the presence of $b l a_{\text {CTX-M-27. }}$. The C1-M27 subclade has previously been responsible for increases in ESBL-producing E. coli from Japan and was also present among ST131 obtained from Thailand, Australia, Canada, and the United States (6). The ST131 C1M27 subclade is currently emerging in Germany (32) and France (33) and is responsible for $27 \%$ of 144 clinical ST131 obtained from different sites in Europe (34).

Clade A is likely the ancestral lineage of ST131 and probably originated in Southeast Asia during the mid to late 1880s (30). Clade A isolates are generally sensitive to antimicrobial drugs and appear to occupy distinct ecologic niches, such as waste water (35). Results from this study show that clade A isolates have high not susceptible rates for trimethoprim/sulfamethoxazole, gentamicin, and tobramycin and were associated with community-associated and healthcare-associated urinary tract infections in younger patients. The virulence factors afa $A B C D$, draABCDP, vat, and traT were common in clade A. Also, clade A was absent among ST131 from 2006 but became the third most common clade during 2012 and 2016, replacing clades B and C0 during these periods.

Clade B emerged from clade A in the early 1900s and most likely occurred in North America $(10,30)$. Members of clade B are antimicrobial susceptible, and several intermediate subclades have been identified (29). Our study showed that clade B isolates were the second most common clade during 2006 but decreased substantially during 2016 . This clade was the most antimicrobial sensitive ST131 clade in Calgary and was associated with community-acquired urinary tract infections and virulence factors $a f a A B C D$, draABCDP, kpsMII, and ibe $A B C$.

Previous data have shown that gyrA S83L mutations occurred first among fluoroquinolone-resistant 
Trends in Population Dynamics of E. coli ST131

Table 4. Virulence factors associated with Escherichia coli sequence type 131 clades, Calgary, Alberta, Canada, 2006, 2012, and $2016^{*}$

\begin{tabular}{|c|c|c|c|c|c|c|c|}
\hline \multirow[b]{2}{*}{ Factor } & \multicolumn{7}{|c|}{ Clade } \\
\hline & $A, n=34$ & $B, n=32$ & $\mathrm{C} 0, \mathrm{n}=5$ & $\begin{array}{c}\text { C1-nonM27, } \\
\mathrm{n}=121\end{array}$ & $\mathrm{C} 1-\mathrm{M} 27, \mathrm{n}=13$ & $\mathrm{C} 2, \mathrm{n}=139$ & All, $n=344$ \\
\hline \multicolumn{8}{|l|}{ Adhesion gene } \\
\hline papA & 34 (100) & $28(88)$ & $5(100)$ & $121(100)$ & 13 (100) & $139(100)$ & 340 (99) \\
\hline papB & $1(3)^{a}$ & $11(34)$ & 0 & $35(29)^{a}$ & $1(8)$ & $90(65)^{b}$ & $138(40)$ \\
\hline papC & $1(3)^{a}$ & $13(41)$ & 0 & $31(26)^{a}$ & $2(16)$ & $89(64)^{b}$ & $141(41)$ \\
\hline papD & $1(3)^{a}$ & $13(41)$ & 0 & $36(30)^{a}$ & $1(8)$ & $89(64)^{b}$ & $140(41)$ \\
\hline papE & $1(3)$ & $11(34)$ & 0 & 0 & 0 & $9(6)$ & $21(6)$ \\
\hline papF & $2(6)^{a}$ & 12 (38) & 0 & $34(28)^{a}$ & $1(8)$ & $90(65)^{\mathrm{b}}$ & $139(40)$ \\
\hline papG & $1(3)$ & $14(44)$ & 0 & $34(28)$ & $1(8)$ & $91(6)$ & $141(41)$ \\
\hline papH & $1(3)$ & $9(28)$ & 0 & 33 (27) & 0 & 41 (29) & $84(24)$ \\
\hline papl & $32(94)$ & $24(75)^{a}$ & $5(100)$ & $116(96)$ & $12(92)$ & $137(99)^{b}$ & $326(95)$ \\
\hline papJ & $1(3)^{a}$ & $11(34)$ & 0 & $34(28)^{a}$ & $1(8)$ & $90(65)^{b}$ & 137 (40) \\
\hline papK & $1(3)^{a}$ & $11(34)$ & 0 & $34(28)^{a}$ & $1(8)$ & $90(65)^{b}$ & 137 (40) \\
\hline papX & $32(94)$ & $21(66)^{a}$ & $5(100)$ & $100(83)$ & $10(77)$ & $134(96)^{b}$ & 302 (88) \\
\hline Iha & 33 (97) & $16(50)^{a}$ & $5(100)$ & $111(92)$ & 13 (100) & $137(99)^{b}$ & 314 (91) \\
\hline $\mathrm{fimH}$ & 34 (100) & 32 (100) & $5(100)$ & $121(100)$ & $13(100)$ & 139 (100) & 344 (100) \\
\hline Tsh & 0 & $2(6)$ & 0 & 0 & 0 & 0 & $2(0.6)$ \\
\hline Hra & $1(1)$ & 0 & $1(25)$ & $1(1)$ & 0 & $8(6)$ & $11(3)$ \\
\hline $\operatorname{afa} A B C D$ & $18(53)^{a}$ & $18(56)^{a}$ & $1(25)$ & $4(3)^{b}$ & 0 & $28(20)^{\mathrm{b}}$ & $69(20)$ \\
\hline draABCDP & $18(53)^{a}$ & $18(56)^{a}$ & $1(25)$ & $4(3)^{b}$ & 0 & $28(20)^{b}$ & $69(20)$ \\
\hline \multicolumn{8}{|l|}{ Toxin gene } \\
\hline hlyA & $1(1)$ & $11(34)$ & 0 & $27(22)$ & 0 & $50(36)$ & $89(26)$ \\
\hline Sat & $30(88)$ & $21(66)^{a}$ & $5(100)$ & $112(93)$ & $13(100)$ & $136(98)^{b}$ & $317(92)$ \\
\hline Vat & $34(100)^{a}$ & $7(22)^{b}$ & 0 & $1(1)^{b}$ & $0^{b}$ & $0^{\mathrm{b}}$ & $42(1)$ \\
\hline ast $A$ & $1(3)$ & $1(3)$ & 0 & 0 & 0 & $6(4)$ & $8(2)$ \\
\hline cnf1 & $1(3)$ & $9(28)$ & 0 & $29(24)$ & 0 & $52(37)$ & $91(26)$ \\
\hline \multicolumn{8}{|l|}{ Siderophore gene } \\
\hline iroN & 0 & $9(28)$ & 0 & $1(1)$ & 0 & $9(6)$ & $19(6)$ \\
\hline fyuA & 34 (100) & $32(100)$ & $5(100)$ & $121(100)$ & $13(100)$ & $139(100)$ & $344(100)$ \\
\hline ireA & 0 & 0 & 0 & 0 & 0 & $11(8)$ & $11(3)$ \\
\hline iutA & $29(85)$ & $27(84)$ & $5(100)$ & $112(93)$ & $12(92)$ & $137(99)$ & $322(94)$ \\
\hline \multicolumn{8}{|l|}{ Capsular antigen gene } \\
\hline$k p s M I I$ & 0 & $14(44)$ & 0 & 0 & 0 & $2(1)$ & $16(5)$ \\
\hline kpsMT III & $0^{a}$ & $13(41)$ & $5(100)$ & $71(59)^{a, b}$ & $13(100)^{b}$ & $37(26)^{c}$ & $139(40)$ \\
\hline \multicolumn{8}{|l|}{ Miscellaneous gene } \\
\hline Usp & $33(97)$ & $32(100)$ & $5(100)$ & $121(100)$ & $12(92)$ & $137(99)$ & 340 (99) \\
\hline trat & $28(82)^{\mathrm{a}}$ & $13(41)^{\mathrm{b}}$ & 0 & $20(17)^{\mathrm{b}}$ & $0^{\mathrm{b}}$ & $28(20)^{b}$ & $89(26)$ \\
\hline ompT & 0 & $4(13)$ & 0 & $2(2)$ & 0 & $1(1)$ & $7(2)$ \\
\hline Iss & $9(26)^{a}$ & $31(97)$ & $5(100)$ & $118(98)^{b}$ & $13(100)$ & $139(100)^{b}$ & $315(92)$ \\
\hline malX & $34(100)$ & $32(100)$ & $5(100)$ & $118(98)$ & $13(100)$ & $138(99)$ & 340 (99) \\
\hline$c d t B$ & 0 & $2(6)$ & 0 & 0 & 0 & 0 & $2(0.6)$ \\
\hline cvaC & 0 & $4(13)$ & 0 & 0 & $9(69 \%)$ & $10(7 \%)$ & $14(6)$ \\
\hline$i b e A B C$ & 0 & $14(44)^{a}$ & 0 & 0 & 0 & $0^{\mathrm{b}}$ & $14(6)$ \\
\hline Virulence score, median (range)† & $11(7-15)$ & $10(6-14)$ & $11(9-16)$ & $10(6-15)$ & $10(9-14)$ & $11(7-15)$ & \\
\hline \multicolumn{8}{|c|}{$\begin{array}{l}\text { *Values are no. (\%) unless indicated otherwise. Rates followed by different superscript letters indicate significant differences between clades at the } 5 \% \\
\text { level (adjusted for multiple comparisons). afa, afimbrial adhesin; astA, enteroaggregative } E \text {. coli toxin; } c d t B \text {, cytolethal distending toxin B; cnf1, cytotoxic } \\
\text { necrotizing factor; cvaC, factor facilitating colonization; dra, Dr binding adhesins; fimH, type-1 fimbriae; fyuA, yersiniabactin (siderophore) receptor; hlyA, } \\
\alpha \text {-hemolysin; hra, heat-resistant agglutinin; ibeABC, invasion of brain endothelium; iha, iron-regulated adhesin; ireA, iron-regulated element (catecholate } \\
\text { siderophore); iroN, salmochelin (siderophore) receptor; iss, increased serum survival; iutA, aerobactin (siderophore) receptor; kpsM II, group II capsule } \\
\text { variants synthesis; kpsM III, group III capsule variants synthesis; malX, pathogenicity island marker; ompT, outer membrane protein T; papA, P fimbriae; } \\
\text { papBCDEFGHIJKX, genes of P fimbriae operon; sat, secreted autotransporter toxin; traT, complement inhibition protein; tsh, temperature-sensitive } \\
\text { hemagglutinin; usp, uropathogenic-specific protein; vat, vacuolating autotransporter toxin. } \\
\text { †The virulence gene score was the number of virulence operons detected. }\end{array}$} \\
\hline
\end{tabular}

E. coli and is a major initial step for establishing relative fitness among antimicrobial-resistant isolates (36). Our study showed that gyrA mutations were rare among clade B isolates, but parE I529L mutations were common. This finding suggests that parE I529L mutations are the first to occur among fluoroquinolone-resistant ST131. The order in which these mutations arise might play a major role in establishing fitness in ST131 (37).
Our study had some limitations. Only patients in Calgary who had positive blood cultures for $E$. coli were included, which excluded those with E. coli bloodstream infections from whom no blood samples were submitted for culture. Therefore, incidence rates should be considered as conservative estimates of ST131 bloodstream infections in Calgary, especially for patients infected with clades A and B, who tended to be younger (i.e., clade $\mathrm{A}$ infections) and from the 
community (i.e., clade B infections). Such patients were less likely to have had blood cultures taken than patients who are older or who had previous contact with the healthcare system.

The novel approach for our study used population-based surveillance to describe the incidence rates, specific characteristics, and trends among ST131 clades over an 11-year period in a well-defined human population. We showed major differences in IRs, frequencies, resistance patterns, antimicrobial resistance determinants, grouped plasmid types, virulence factors, and trends over time for different clades. We provided insights into the evolution of ST131 clades in a large well-defined region of Canada. The population structure of ST131 in large geographic healthcare regions is dynamic and has continuous interplay between different subclades.

A previous study showed that eliminating ST131 would substantially decrease the overall IR and antimicrobial-resistant burden within E. coli causing bloodstream infections in the Calgary region (7). This study identified ST131 subclade C2 as the predominant and most antimicrobial-resistant subclade in Calgary, which is increasing exponentially over time. Eradicating ST131, more specifically the C2 subclade, will lead to considerable public health benefits for persons in Calgary.

This study was supported by research grant \#10016015 from the Joint Programming Initiative on Antimicrobial Resistance/Canadian Institute Health Research Program.

\section{About the Author}

Dr. Peirano is a research associate at Alberta Precision Laboratories and the University of Calgary, Calgary, Alberta, Canada. Her research interests include the molecular epidemiology of antimicrobial drugresistant organisms.

\section{References}

1. Mathers AJ, Peirano G, Pitout JD. The role of epidemic resistance plasmids and international high-risk clones in the spread of multidrug-resistant Enterobacteriaceae. Clin Microbiol Rev. 2015;28:565-91. https:/ / doi.org/10.1128/CMR.00116-14

2. Peirano G, Pitout JD. Molecular epidemiology of Escherichia coli producing CTX-M beta-lactamases: the worldwide emergence of clone ST131 O25:H4. Int J Antimicrob Agents. 2010;35:316-21. https://doi.org/ 10.1016/j.ijantimicag.2009.11.003

3. Manges AR, Geum HM, Guo A, Edens TJ, Fibke CD, Pitout J. Global extraintestinal pathogenic Escherichia coli (ExPEC) lineages. Clin Microbiol Rev. 2019;32:e00135-18. https://doi.org/10.1128/CMR.00135-18

4. Peirano G, Pitout JDD. Extended-spectrum $\beta$-lactamaseproducing Enterobacteriaceae: update on molecular epidemiology and treatment options. Drugs. 2019;79:152941. https:/ / doi.org/10.1007/s40265-019-01180-3

5. Pitout JD, DeVinney R. Escherichia coli ST131: a multidrugresistant clone primed for global domination. F1000 Res. 2017;6:6. https://doi.org/10.12688/f1000research.10609.1

6. Matsumura Y, Pitout JD, Gomi R, Matsuda T, Noguchi T, Yamamoto M, et al. Global Escherichia coli sequence type 131 clade with bla ${ }_{\mathrm{CTX}-\mathrm{M}-27}$ gene. Emerg Infect Dis. 2016;22:1900-7. https:// doi.org/10.3201/eid2211.160519

7. Holland MS, Nobrega D, Peirano G, Naugler C, Church DL, Pitout JDD. Molecular epidemiology of Escherichia coli causing bloodstream infections in a centralized Canadian region: a population-based surveillance study. Clin Microbiol Infect. 2020;S1198-743X(20)30101-4. https:/ / doi.org/10.1016/j.cmi.2020.02.019

8. Peirano G, Pitout JD. Fluoroquinolone-resistant Escherichia coli sequence type 131 isolates causing bloodstream infections in a Canadian region with a centralized laboratory system: rapid emergence of the H30-Rx sublineage. Antimicrob Agents Chemother. 2014;58:2699-703. https:/ / doi.org/10.1128/AAC.00119-14

9. Peirano G, van der Bij AK, Gregson DB, Pitout JD. Molecular epidemiology over an 11-year period (2000 to 2010) of extended-spectrum $\beta$-lactamase-producing Escherichia coli causing bacteremia in a centralized Canadian region. J Clin Microbiol. 2012;50:294-9. https://doi.org/10.1128/ JCM.06025-11

10. Pitout JDD, Finn TJ. The evolutionary puzzle of Escherichia coli ST131. Infect Genet Evol. 2020;81:104265. https:/ / doi.org/10.1016/j.meegid.2020.104265

11. Friedman ND, Kaye KS, Stout JE, McGarry SA, Trivette SL, Briggs JP, et al. Health care-associated bloodstream infections in adults: a reason to change the accepted definition of community-acquired infections. Ann Intern Med. 2002;137:791-7. https:/ / doi.org/10.7326/00034819-137-10-200211190-00007

12. Clinical and Laboratory Standards Institute. Perfomance standards for antimicrobial susceptibility testing. 25th information supplement. CLSI document M100-S25. Wayne (PA): The Institute; 2015.

13. Johnson JR, Porter $S$, Thuras P, Castanheira M. The pandemic H30 subclone of sequence type 131 (ST131) as the leading cause of multidrug-resistant Escherichia coli infections in the United States (2011-2012). Open Forum Infect Dis. 2017;4:ofx089. https://doi.org/10.1093/ofid/ofx089

14. Matsumura Y, Pitout JD, Peirano G, DeVinney R, Noguchi T, Yamamoto M, et al. Rapidildentification of different Escherichia coli sequence type 131 clades. Antimicrob Agents Chemother. 2017;61:e00179-17. https:/ / doi.org/10.1128/AAC.00179-17

15. Lowe M, Kock MM, Coetzee J, Hoosien E, Peirano G, Strydom KA, et al. Klebsiella pneumoniae ST307 with bla ${ }_{\mathrm{OXA}_{\mathrm{A} 181}}$ South Africa, 2014-2016. Emerg Infect Dis. 2019;25:739-47. https://doi.org/10.3201/eid2504.181482

16. Peirano G, Matsumura Y, Adams MD, Bradford P, Motyl M, Chen L, et al. Genomic epidemiology of global carbapenemase-producing Enterobacter spp., 2008-2014. Emerg Infect Dis. 2018;24:1010-9. https:// doi.org/10.3201/ eid2406.171648

17. Nurk S, Bankevich A, Antipov D, Gurevich AA, Korobeynikov A, Lapidus A, et al. Assembling single-cell genomes and mini-metagenomes from chimeric MDA products. J Comput Biol. 2013;20:714-37. https:/ / doi.org/ $10.1089 / \mathrm{cmb} .2013 .0084$

18. Camacho C, Coulouris G, Avagyan V, Ma N, Papadopoulos J, Bealer K, et al. BLAST+: architecture and applications. 
BMC Bioinformatics. 2009;10:421. https:// doi.org/10.1186/ 1471-2105-10-421

19. Zankari E, Hasman H, Cosentino S, Vestergaard M, Rasmussen S, Lund O, et al. Identification of acquired antimicrobial resistance genes. J Antimicrob Chemother. 2012;67:2640-4. https://doi.org/10.1093/jac/dks261

20. Carattoli A, Zankari E, García-Fernández A, Voldby Larsen M, Lund $\mathrm{O}$, Villa L, et al. In silico detection and typing of plasmids using PlasmidFinder and plasmid multilocus sequence typing. Antimicrob Agents Chemother. 2014;58:3895-903. https://doi.org/10.1128/ AAC.02412-14

21. Zhou Z, Alikhan NF, Mohamed K, Fan Y, Achtman M; Agama Study Group. The EnteroBase user's guide, with case studies on Salmonella transmissions, Yersinia pestis phylogeny, and Escherichia core genomic diversity. Genome Res. 2020;30:138-52. https:/ / doi.org/10.1101/gr.251678.119

22. Joensen KG, Scheutz F, Lund O, Hasman H, Kaas RS, Nielsen EM, et al. Real-time whole-genome sequencing for routine typing, surveillance, and outbreak detection of verotoxigenic Escherichia coli. J Clin Microbiol. 2014;52:150110. https://doi.org/10.1128/JCM.03617-13

23. Liu B, Zheng D, Jin Q, Chen L, Yang J. VFDB 2019: a comparative pathogenomic platform with an interactive web interface. Nucleic Acids Res. 2019;47(D1):D687-92. https://doi.org/10.1093/nar/gky1080

24. Benjamini Y, Hochberg Y. Controlling the false discovery rate: a practical and powerful approach to multiple testing. J R Stat Soc B. 1995;57:289-300. https:// doi.org/10.1111/ j.2517-6161.1995.tb02031.x

25. R Core Team. R: a language and environment for statistical computing. Vienna: R Foundation for Statistical Computing; 2017.

26. Kondratyeva K, Salmon-Divon M, Navon-Venezia S. Meta-analysis of pandemic Escherichia coli ST131 plasmidome proves restricted plasmid-clade associations. Sci Rep. 2020;10:36. https:/ / doi.org/10.1038/s41598-019-56763-7

27. Hughes JM, Lohman BK, Deckert GE, Nichols EP, Settles M, Abdo Z, et al. The role of clonal interference in the evolutionary dynamics of plasmid-host adaptation. MBio. 2012;3:e00077-12. https://doi.org/10.1128/mBio.00077-12

28. Decano AG, Downing T. An Escherichia coli ST131 pangenome atlas reveals population structure and evolution across 4,071 isolates. Sci Rep. 2019;9:17394. https:/ / doi.org/ 10.1038/s41598-019-54004-5

29. Ben Zakour NL, Alsheikh-Hussain AS, Ashcroft MM, Khanh Nhu NT, Roberts LW, Stanton-Cook M, et al. Sequential acquisition of virulence and fluoroquinolone resistance has shaped the evolution of Escherichia coli ST131. mBio. 2016;7:e00347-16.
30. Stoesser N, Sheppard AE, Pankhurst L, De Maio N, Moore CE, Sebra R, et al.; Modernizing Medical Microbiology Informatics Group (MMMIG). Evolutionary history of the global emergence of the Escherichia coli epidemic clone ST131. MBio. 2016;7:e02162. https://doi.org/10.1128/ mBio.02162-15

31. Galtier M, De Sordi L, Maura D, Arachchi H, Volant S, Dillies MA, et al. Bacteriophages to reduce gut carriage of antibiotic resistant uropathogens with low impact on microbiota composition. Environ Microbiol. 2016;18:2237-45. https:// doi.org/10.1111/1462-2920.13284

32. Ghosh H, Doijad S, Falgenhauer L, Fritzenwanker M, Imirzalioglu C, Chakraborty T. bla ${ }_{\text {CTX-M-27 }}$-encoding Escherichia coli sequence type 131 lineage C1-M27 clone in clinical isolates, Germany. Emerg Infect Dis. 2017;23:1754-6. https://doi.org/10.3201/eid2310.170938

33. Birgy A, Bidet P, Levy C, Sobral E, Cohen R, Bonacorsi S. CTX-M-27-producing Escherichia coli of sequence type 131 and clade C1-M27, France. Emerg Infect Dis. 2017;23:885. https://doi.org/10.3201/eid2305.161865

34. Merino I, Hernández-García M, Turrientes MC, Pérez-Viso B, López-Fresneña N, Diaz-Agero C, et al.; R-GNOSIS Study Group. Emergence of ESBL-producing Escherichia coli ST131-C1-M27 clade colonizing patients in Europe. J Antimicrob Chemother. 2018;73:2973-80. https:/ / doi.org/ 10.1093/jac/dky296

35. Finn TJ, Scriver L, Lam L, Duong M, Peirano G, Lynch T, et al. A comprehensive account of Escherichia coli sequence type 131 in wastewater reveals an abundance of fluoroquinoloneresistant clade A strains. Appl Environ Microbiol. 2020; 86:e01913-19. https://doi.org/10.1128/AEM.01913-19

36. Huseby DL, Pietsch F, Brandis G, Garoff L, Tegehall A, Hughes D. Mutation supply and relative fitness shape the genotypes of ciprofloxacin-resistant Escherichia coli. Mol Biol Evol. 2017;34:1029-39. https:// doi.org/10.1093/molbev/ msx052

37. Johnson JR, Johnston B, Kuskowski MA, Sokurenko EV, Tchesnokova V. Intensity and mechanisms of fluoroquinolone resistance within the $\mathrm{H} 30$ and $\mathrm{H} 30 \mathrm{Rx}$ subclones of Escherichia coli sequence type 131 compared with other fluoroquinoloneresistant E. coli. Antimicrob Agents Chemother. 2015;59:447180. https://doi.org/10.1128/AAC.00673-15

Address for correspondence: Johann D.D. Pitout, Department of Pathology and Laboratory Medicine, University of Calgary, \#9 3535 Research Rd NW, Calgary T2L 2K8, Alberta, Canada; email: jpitout@ucalgary.ca 